\title{
In-Network Processing for Low-Latency Industrial Anomaly Detection in Softwarized Networks
}

\author{
Huanzhuo Wu*, Jia He*, Máté Tömösközi*†, Zuo Xiang* and Frank H.P. Fitzek*† \\ ${ }^{*}$ Deutsche Telekom Chair of Communication Networks - Technische Universität Dresden, Germany \\ ${ }^{\dagger}$ Centre for Tactile Internet with Human-in-the-Loop (CeTI) \\ Email: $\{$ huanzhuo.wu|mate.tomoskozi|zuo.xiang|frank.fitzek\}@tu-dresden.de, jia.he@mailbox.tu-dresden.de
}

\begin{abstract}
Modern manufacturers are currently undertaking the integration of novel digital technologies - such as 5Gbased wireless networks, the Internet of Things (IoT), and cloud computing - to elevate their production process to a brand new level, the level of smart factories. In the setting of a modern smart factory, time-critical applications are increasingly important to facilitate efficient and safe production. However, these applications suffer from delays in data transmission and processing due to the high density of wireless sensors and the large volumes of data that they generate. As the advent of nextgeneration networks has made network nodes intelligent and capable of handling multiple network functions, the increased computational power of the nodes makes it possible to offload some of the computational overhead. In this paper, we show for the first time our IA-Net-Lite industrial anomaly detection system with the novel capability of in-network data processing. IA-Net-Lite utilizes intelligent network devices to combine data transmission and processing, as well as to progressively filter redundant data in order to optimize service latency. By testing in a practical network emulator, we showed that the proposed approach can reduce the service latency by up to $40 \%$. Moreover, the benefits of our approach could potentially be exploited in other large-volume and artificial intelligence applications.
\end{abstract}

Index Terms - anomaly detection, in-network computing, network softwarization, internet of things

\section{INTRODUCTION}

Presently, the general industrial community is facing the challenge of integrating Industry 4.0 technologies into their manufacturing processes, which will eventually lead towards full digitization and intelligence empowered by such emerging technologies as Internet of Things (IoT), artificial intelligence (AI), and cloud computing [2]. Among these, $[$ IoT devices are expected to act as both data collectors and the actuators of remote intelligent computing agents, thereby closing the full control loop in order to realize a fully autonomous factory operation. In future smart factories, IoT devices would be tasked with the monitoring of processes and machines, etc. The data produced by such IoT devices can be, in turn, employed to detect potential disturbances in the production process before an error happens (predictive maintenance), which can decrease production downtime and maintenance costs of smart factories [3].

In particular, time-critical acoustic anomaly detection in industrial environments carries great potential, since acoustic

This is a preprint of the work [1], that has been accepted for publication in the proceedings of the 2021 IEEE Global Communications Conference. data is able to foreshadow potential failures in the earlystage and offers significant gains to accurately predict when maintenance work is crucial [4]. Unlike traditional industrial signals - e.g., temperature and humidity - acoustic signals are generally distorted before an abnormal event occurs. Also, such audio-based detection methods can avoid blocking and angular distortion problems compared to the alternative image analysis techniques, as well.

IoT-enabled acoustic sensors deployed in factory halls normally collect and transmit the raw sensor readings for further analysis to separate dedicated devices. In a generic setup, several devices are deployed at the production site, which creates a multi-object acoustic anomaly detection environment. Particularly, the observed raw sensory acoustic signal is inevitably altered (mixed) due to the presence and operation of multiple objects. Depending on the complexity of the deployed sensors and observation systems, the overall amount of data generated can easily reach several Gbps ranges, which can be understood as a big data problem with data-rich, information-poor characteristics [5]. In [6], a data process based on a Deep Neural Network (DNN) and Information Abstraction Neural Network (IA-Net) is proposed to address the challenge of data-rich, informationpoor environments, which empowers detection techniques with high-information density (the acoustic deviation). After IA-Net solved the issue of processing large amounts of IoT data for anomaly detection, we are now facing the question of where should IA-Net do the processing?

The most straightforward solution is to employ a centralized service that manages the handling of the data stemming from the growing volume of sensors. This effectiveness relies on the local Internet access, which is often limited to a few hundred Mbps. While large companies can simply upgrade their infrastructure, most small and mid-sized industrial manufacturers rely on traditional ISPs for their networking needs, and higher access speeds are tied to higher costs [7]. A different approach would be to deploy IA-Net on an edge cloud physically close to the targeted machines. Compared with the centralized approach, the edge cloud has an advantage in data transmission time, however, its resource-constrained characteristics will lead to more delay in data processing and, ultimately, would prevent the fulfillment of time-critical services.

With the aid of network softwarization, it is possible 
to deploy flexible services and applications by combining local, edge, and remote computing resources within the same logical network [8]. Typically, the deployment of softwarized networks includes a combination of technologies: Software Defined Networking (SDN) [9], Network Functions Virtualization (NFV) [10], and Service Function Chain (SFC) [11]. Utilizing available computational resources within the network for in-network processing concepts, computation steps can be deployed in the on-premise network to reduce the overall data volume and, potentially, speed up processing times [12]. Therefore, a major challenge is to filter out redundant data by applying the processing logic of information abstraction on resource-constrained network nodes.

In this paper, based on the idea of information abstraction proposed in [6], we show the novel design of a lightweight DNN model called Information Abstraction Neural Network Lite (IA-Net-Lite), and propose its deployment for the first time in softwarized networks, which, contrary to present norms, enables innetwork data processing to achieve low-latency industrial anomaly detection. Extensive evaluations of practical implementations show that IA-Net-Lite reduces the computation load by up to a quarter of the original and decreases the data transfer delays by about $34.38 \%$, thus achieving an overall latency reduction of up to $38.89 \%$.

The rest of the paper is structured as follows. First, Section [II introduces the idea of information abstraction and describes the problem, after which the proposed method is described in Section [III In Section IV] we introduce the experimental setup and its metrics. Section $\mathrm{V}$ covers the numerical evaluation and a discussion about our measurement results. Finally, Section VI concludes our contribution and points out future research aspects.

\section{BACKGROUND}

In this section, we first describe our system for acoustic anomaly detection and the methods used to filter out redundant information. Following that, we discuss the importance of this redundant information filtering approach for low-latency services and the challenges when exploiting it in networks.

\section{A. Information Abstraction}

In multi-machine environments, such as factories, a number of source machines $(n)$ are expected to be detected as source objects, see Fig. 10 The source of acoustic data is generated in $m$ time slots and is denoted as $\left\{\mathbf{s}_{1}, \mathbf{s}_{2}, \cdots, \mathbf{s}_{n}\right\}=\mathbf{S} \in \mathbb{R}^{n \times m}$. Due to the presence and operation of multiple objects, the observation $\mathbf{X} \in \mathbb{R}^{1 \times m}$ of IoT sensors is inevitably a mixture of the $n$ source data. $\mathbf{X}$, in turn, is transmitted to the data processing system to determine irregularities (or anomalies) related to the $n$ objects when only $\mathbf{X}$ is known.

Such an anomaly is defined by the anomaly score and is denoted as $\mathcal{A}$. $\mathcal{A}$ represents the difference between the actual data and a reference $\mathbf{S}^{r}$. When $\mathcal{A}$ exceeds a threshold value, the corresponding object is declared to be an anomaly, as

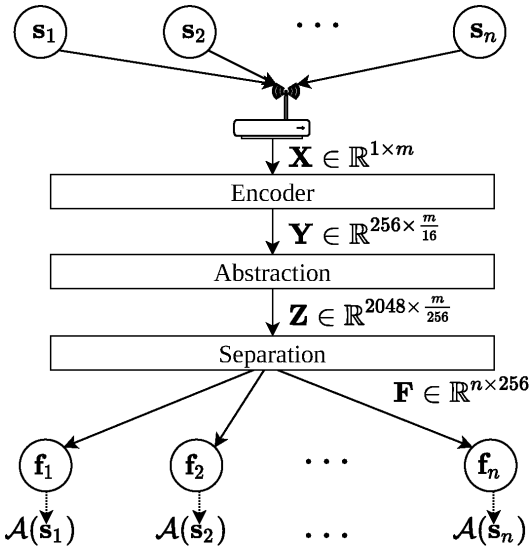

Fig. 1: The schematics of information abstraction for industrial anomaly detection.

shown in Fig. 1 In order to get $\mathcal{A}$, more attention needs to be paid to the deviation between the detected and the reference objects than to the detected object itself. Therefore, redundant information not related to exceptions should be eliminated and only exception-related information needs to be extracted. Based on this intuition, we proposed in [6] the idea of information abstraction to abstract separated anomalous information from raw sensory data for multi-object acoustic anomaly detection, which is a particularly advantageous data processing approach when handling large data volumes.

As shown in Fig. 1, the information abstraction consists of three distinct modules: encoder, abstraction, and separation. The encoder converts $\mathbf{X}$ from the sensor into high-dimensional representations $\mathbf{Y} \in \mathbb{R}^{256 \times \frac{m}{16}}$ to provide more detailed information. Then abstraction retrieves the features $\mathbf{Z} \in \mathbb{R}^{2048 \times \frac{m}{256}}$ related to anomalies, and finally the separation pairs $\mathbf{Z}$ to each $i$-th machine $\mathbf{f}_{i} \in \mathbb{R}^{1 \times 256}$. Based on these anomaly features, the anomaly score can be obtained directly.

\section{B. In-Network (Pre-)Processing}

As a DNN model, the processing logic of information abstraction is often deployed centralized on a dedicated server/cloud due to the large computational requirements. However, with the centralized deployment, all raw sensory data $\mathbf{X}$ needs to be transmitted through the network to this server/cloud, i.e., the entire sequence of the generated audio of each client. Such a large volume of data will inevitably lead to network congestion and data transmission delays.

An alternative way is to deploy the processing logic on an edge cloud near the individual [oT sensors in order to achieve shorter transmission delays. However, due to the nature of networks, the computational resources (in this case, processing and memory) are limited on edge nodes. This limitation increases the processing delay, which in effect results in limited or no reduction in the total service latency. As an example, $9.08 \times 10^{9}$ operations are needed by [A-Net [6] to complete the processing task.

Fortunately, information abstraction can eliminate redundant information. With the development of network softwarization, 


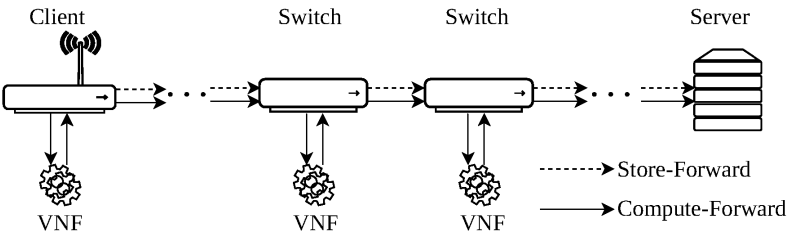

Fig. 2: Overview of the in-network data processing scenario.

computational resources in the network become feasible for performing intermediate processing. Therefore, we can consider that the entire processing logic is partitioned into several Virtual Network Functions (VNFs) that filter redundant data one by one. When these VNF; consume only a small amount of computational resources, the resource-constrained network nodes can perform partial data processing tasks without adding additional processing latency. At the same time, through these VNF;, redundant data can be gradually filtered out without causing network congestion and increased transmission latency. The nodes in the network also collaborate toward optimizing the overall service latency.

Therefore, following the above idea, this paper takes industrial anomaly detection as a case study to solve the following two problems:

1) How to design the processing logic for the resourceconstrained network nodes so that it is deployable as a $\mathrm{VNF}$ without extra processing delay?

2) How should such a VNF be deployed in the network to achieve the purpose of redundant data filtering?

\section{Our Solution: IA-Net-Lite}

In this section, we first show the design of our lightweight DNN model-based Information Abstraction Neural Network Lite (IA-Net-Lite, so that it is suitable for deployment on resource-constrained network nodes in order to abstract information. Secondly, we discuss how it should be partitioned and deployed to filter traffic to optimize service latency.

Fig. 2 illustrates the scenario of in-network data processing. The collected data is constantly sent to a network client, where the client can be either a WiFi access point or a cellular base station of a (non-public) network. This client keeps forwarding the data to the backend "server" over a forwarding path. This path can be either dynamically determined based on a routing mechanism or statically configured. The forwarding path consists of some intermediate network switches, which support SDN and NFV

In a traditional communication network, the network nodes (i.e., clients and switches) are only responsible for forwarding data to the server, and the entire DNN model is deployed on the server to process the forwarded data. This approach is usually called Store-and-Forward (S-F) In the scenario of the in-network process, the DNN model is split into several suboperations, which are deployed as VNF on the network nodes along the data path. These network nodes perform more than just forwarding data: they first process the data using the VNF

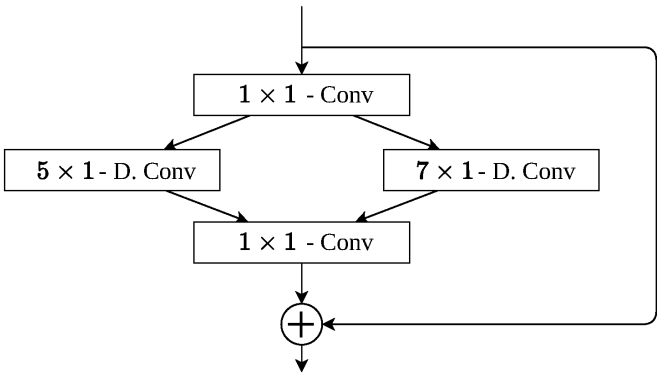

Fig. 3: 1D-residual convolution (1D-R-Conv).

and then forward the intermediate results to the next node. This is commonly known as Compute-and-Forward (C-F).

\section{A. A Lightweight Deep Neural Network Model for Networks}

As the resources (computing and storage) of network devices are limited, the overall parameters and operations of a DNN model should be tightly controlled at an acceptable scale, which is suitable for such lightweight devices. The complexity of a DNN model during inference is defined by the memory and computing power required to process the input data. This can be mathematically formulated by the number of the parameters (Param) and the number of the Multiply-Add Operations (MAdds)

To minimize Param and MAdd we designed IA-Net-Lite for in-network processing, inspired by IA-Net in [6]. Under the guidance of the paradigm from IA-Net IA-Net-Lite also consists of three distinct modules: encoder, abstraction, and separation.

1) Encoder: In the encoder module, the one-dimensional input data is transformed into a higher dimensional representation, in order to provide more detailed information for subsequent operations. IA-Net-Lite down-samples and maps the mixed input data $\mathbf{X} \in \mathbb{R}^{1 \times m}$ in a high-dimensional STFTlike feature space $\mathbf{Y} \in \mathcal{R}^{N \times \frac{m}{4}}$ by using a 1-D convolutional layer (1D-Conv) with stride four. The 1D-Conv is a set of filters consisting of $N$ filters with length $L$. In the encoder module, only one convolutional layer is used to minimize computation and storage resources. This module eventually only contains $0.18 \times 10^{3}$ Params, and $3.00 \times 10^{6}$ MAdds.

2) Abstraction: The objective of this module is to extract anomaly information $\mathbf{F}$ from the encoded features $\mathbf{Y}$. The abstract module accounts for most of the parameters, which is the main part of the model to be compressed. Therefore, we propose a 1D-residual convolution (1D-R-Conv). As illustrated in Fig. 3, it follows three strategies: $(i)$ depth-wise residual block [13] to reduce the computation; (ii) a large downsampling rate of four to squeeze the input data; (iii) a dual path [14] structure to obtain enriched semantic and temporal domain information and to obtain a balance between accuracy and size.

Empirically, the commonly used $3 \times 1$ convolution kernel causes information loss when the down-sampling rate is four, e.g., ResNet [15]. To avoid information loss during information abstraction while increasing the receptive field of 
TABLE I: The traffic filter of each operations block in IA-Net-Lite.

\begin{tabular}{c|c|c|c}
\hline Operation Block & Module & Input & Output \\
\hline Encoder & 1D-Conv & $1 \times m$ & $32 \times \frac{m}{4}$ \\
Layer 1 & 1D-R-Conv $\times 1$ & $32 \times \frac{m}{4}$ & $16 \times \frac{m}{4}$ \\
Layer 2 & 1D-R-Conv $\times 2$ & $16 \times \frac{m}{4}$ & $24 \times \frac{m}{16}$ \\
Layer 3 & 1D-R-Conv $\times 3$ & $24 \times \frac{m}{16}$ & $32 \times \frac{m}{64}$ \\
Layer 4 & 1D-R-Conv $\times 4$ & $32 \times \frac{m}{64}$ & $64 \times \frac{m}{256}$ \\
Layer 5 & 1D-R-Conv $\times 3$ & $64 \times \frac{m}{256}$ & $96 \times \frac{m}{256}$ \\
Layer 6 & 1D-R-Conv $\times 3$ & $96 \times \frac{m}{256}$ & $160 \times \frac{m}{1024}$ \\
Layer 7 & 1D-R-Conv $\times 1$ & $160 \times \frac{m}{1024}$ & $320 \times \frac{m}{1024}$ \\
Layer 8 & 1D-Conv & $320 \times \frac{m}{1024}$ & $1280 \times \frac{m}{1024}$ \\
Separation & Avg.1D-Conv & $1280 \times \frac{m}{1024}$ & $4 \times 256$ \\
\hline
\end{tabular}

the convolutional layer, we use a large convolutional kernel $7 \times 1$ and $5 \times 1$ in the dual path structure. Each convolution operation is followed by a non-linearity layer (ReLU) and a layer normalization (Norm). The abstraction module consists of several stacked 1D-R-Conv blocks. Although the abstraction module is most resource-intensive, with the lightweight design it only requires $2.29 \times 10^{6}$ Params and $416.00 \times 10^{6}$ MAdds.

3) Decoder: In the decoder module we utilize an average pooling layer (Avg.) to squeeze the temporal information to 1 , and replace the fully-connected layer in the original IANet with a $1 \times 1$ convolutional layer (1D-Conv) to assign the anomalous representations to $n$ objects as abstracted features $\mathbf{F} \in \mathbb{R}^{n \times 256}$. By reducing the number of parameters, the decoder module has $1.31 \times 10^{6}$ Params and $420.00 \times 10^{6}$ MAdds, which is more conducive to deployment in the network.

\section{B. Deployment in the Network}

In a network, usually more data is transmitted than is needed or expected. Therefore, one solution for reducing the amount of data is to use an information filtering logic as a VNF that can be deployed on the network nodes to filter out redundant or unwanted data before the traffic leaves each node.

The limitations on the available resources (processing and memory) of the network nodes is addressed by the lightweight designed DNN model IA-Net-Lite in Section III-A Therefore, compute resources inside the network are available and intermediate processing can be performed in the network and the operation blocks of IA-Net-Lite can be deployed as VNF within the network. However, the question of how computing and networking are integrated, i.e., which operation blocks the deployed VNF should be composed of, is intuitively important. A proper design will reduce the data volume at each hop, consequently, preventing network congestion, and, ultimately, improving the overall service latency.

In Table I the different operation block outputs in relation to the input data for IA-Net-Lite is listed. To better assess the ability of traffic filtering of the different operation blocks, we define filter rate $r$ as follows:

$$
r=\frac{\text { Size of Operation Block Output }}{\text { Size of System Input }}
$$

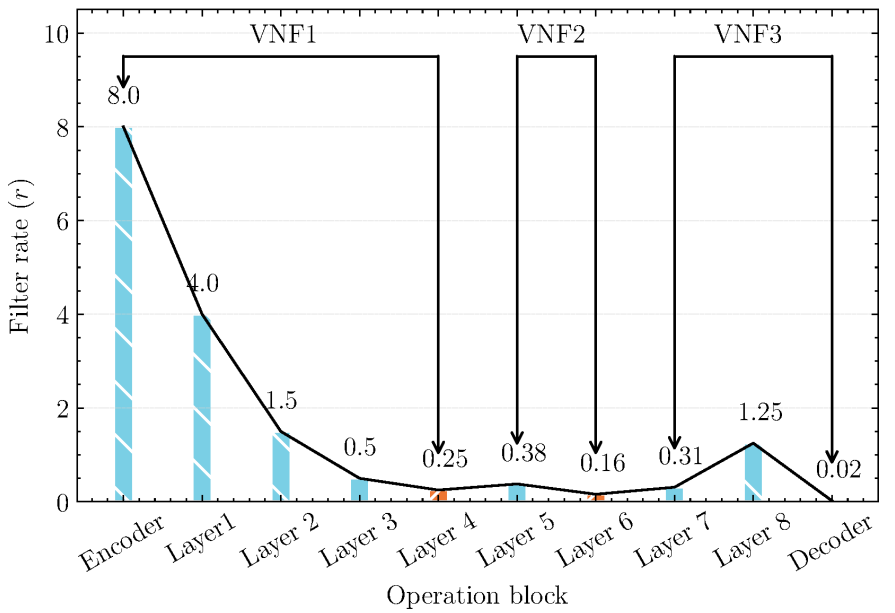

Fig. 4: Filter rate $r$ of data flows by each operation block and the splitting of the VNFs

which indicates how much data has been filtered out by the operation block. An $r>1$ means that the input data is augmented, and vice versa.

The trend of traffic filtering efficiency is fitted to the $r$ of all operation blocks together. To maximize the filtering efficiency on every link in the network, we select the concave points in this filtering trend as the bounds for constructing the VNFs. The concave point of the $r$-curve is represented by the last operation of the continuous data reduction. Splitting before this concave point does not maximize the filtering effect, however, splitting after this point will cause the filtering effect to deteriorate.

As shown in Fig. 4, the $r$ of Layer 4 and Layer 6 are two concave points, which split IA-Net-Lite into three VNFs. By these three VNF; , the data volume can be reduced to $25 \%$, $16 \%$, and $2 \%$ of the original input data, respectively. Therefore, the overall service latency can be improved when these three VNF are deployed on network nodes. In Section V-A we show the evaluation of the effectiveness of traffic filtering.

\section{EXPERIMENTAL DESIGN}

In this section, we describe our experimental design, including the emulation setups and measurement metrics.

\section{A. Emulation Setups}

In our evaluation, we use the realistic dataset of MIMII [16], which is a widely used acoustic data set for malfunctioning industrial machine inspection, containing 26092 normal and anomalous operating acoustic segments from four types of real machinery: valves, pumps, fans, and slide rails. Each acoustic segment is a single-channel 10 -second-long acoustic segment with a sampling rate of $16000 \mathrm{~Hz}$. We randomly select all $n=4$ types of sources from the dataset to construct the source signals $\mathbf{S}$. We mixed the source signals $\mathbf{S}$ following the standard normal distribution to simulate as many mixing scenarios as possible. By doing this, our evaluation has covered various data types and mixing cases. 
We conducted the evaluation on the network emulator Communication Networks Emulator (ComNetsEmu) [17]. As illustrated in Fig. 2, we set up a multi-hop topology with switches as intermediate network nodes. Each node can perform either one or both operations of forwarding and data processing. Hence, there are two different modes for the deployment of the anomaly detection DNN models, which are Compute-and-Forward (C-F) and Store-and-Forward (S-F).

We assume that the clients connect to the network and send the observed data using User Datagram Protocol (UDP) to the server. All topology links have the same homogeneous bandwidth of $10 \mathrm{Mbps}$ and a fixed propagation delay of $150 \mathrm{~ms}$. In the emulation, we do not consider packet losses and assume that all packets are received sequentially. These settings can be considered typical for an edge network. We also assume that all devices and connections are general-purpose and available commercially as is. We implement the client and server functions in Python. For each configuration of a parameter set, we performed 60 measurements in which the client sends the observed data to the server, to ensure the experimental results being statistically significant.

Each packet is forwarded directly to the server by the network node at the fastest possible S-F speed in conventional S-F mode. When operating in the $\mathrm{C}-\mathrm{F}$ mode, the SDN controller inserts rules into the flow table of each switch to forward the data traffic through each middlebox to build an SFC For the overall experiments, we employ a Commercial off-the-shelf (COTS) server with a 2.0G Intel i5 CPU with 16GB RAM using Ubuntu 18.04 LTS.

\section{B. Metrics}

For the evaluation of our method we employ the following metrics:

1) Service Latency: Service latency $t_{s}$ is the Round Trip Time (RTT) between the request sent by the client and the response delivered by the server. It includes all transmission and computation delays introduced by end nodes (client and server) and network nodes. $t_{s}$ greatly affects the QoS of network services. We compare $t_{s}$ of IA-Net-Lite in $\mathrm{C}-\mathrm{F}$ and $\mathrm{S}-\mathrm{F}$ modes, and IA-Net in $\mathrm{S}-\mathrm{F}$ mode.

2) Computation Load: To measure the computation load of an VNF we introduce the metric of processing latency $t_{p}$, which is the computation time required by a device to complete the VNF deployed on it. With the same computational power, a smaller $t_{p}$ means that the VNF on the device is completed faster. As a result, the computation load of this VNF is small. Conversely, a larger $t_{p}$ implies a large computational load for this task.

3) Transmission Latency: Transmission latency $t_{t}$ is the time taken by the network to transmit the packets over the links, including propagation delay, queuing delay, and transmission delay. For the same network setup - e.g., bandwidth, mac access, and so on $-t_{t}$ is closely related to the size of data transmitted on the links.

Overall, in our experimental setups one can assume that $t_{s}=t_{p}+t_{t}$.

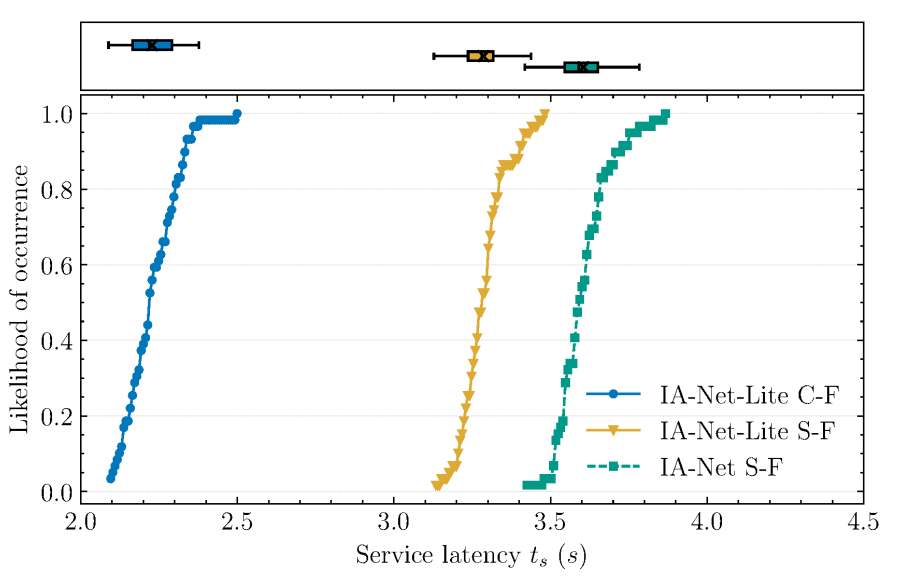

Fig. 5: Likelihood of end-to-end service latency in $\mathrm{C}-\mathrm{F}$ and $\mathrm{S}-\mathrm{F}$ modes.

\section{RESUlTS AND Discussions}

Based on practical experimental results from emulation, we evaluate and discuss the performance of the in-network processing method for low-latency industrial anomaly detection.

\section{A. Service Latency}

Fig. 5] shows the service latency of IA-Net-Lite in C-F and $\mathrm{S}-\mathrm{F}$ modes, and IA-Net in S-F mode, respectively. We observed that in $\mathrm{S}-\mathrm{F}$ mode, the median service latency is reduced from $3.6 \mathrm{~s}$ in IA-Net to $3.3 \mathrm{~s}$ in IA-Net-Lite, which is a $8.33 \%$ reduction. This decrease is due to the fact that our lightweight design makes IA-Net-Lite consume much less computational resources, resulting in less processing latency on the server. Section $\mathrm{V}$-B discusses the lightweight DNN in detail.

More importantly, comparing the median service latency of IA-Net-Lite in C-F and S-F mode, this latency is significantly reduced from $3.3 \mathrm{~s}$ to $2.2 \mathrm{~s}$. This is due to the collaboration of network nodes when filtering out redundant information and thus reducing the transmission latency. In other words, the service latency is reduced by $33.33 \%$ in the in-network processing scheme. Compared to the baseline system IA-Net, this latency is significantly reduced by $38.89 \%$. We discuss traffic filtering in the network in more detail in Section $\mathrm{V}-\mathrm{C}$

To summarize, we propose IA-Net-Lite in this paper for innetwork processing, which can significantly decrease the service latency as it is capable of reducing both the transmission and processing latency with the help of network nodes.

\section{B. Processing Latency and Lightweight Design}

Fig. 6 shows the processing latency of the proposed IA-Net-Lite and the baseline system IA-Net to verify the lightweight design of IA-Net-Lite. In S-F mode, we first observe that the processing latency of IA-Net-Lite is about $0.1 \mathrm{~s}$, which is a mere quarter of IA-Net s. On the one hand, the reduction of the overall processing latency explains the gap between IA-Net-Lite and IA-Net with the S-F mode in Fig. 5 , Moreover, while the server remains the same, reducing the processing latency by quarter means that the computation 


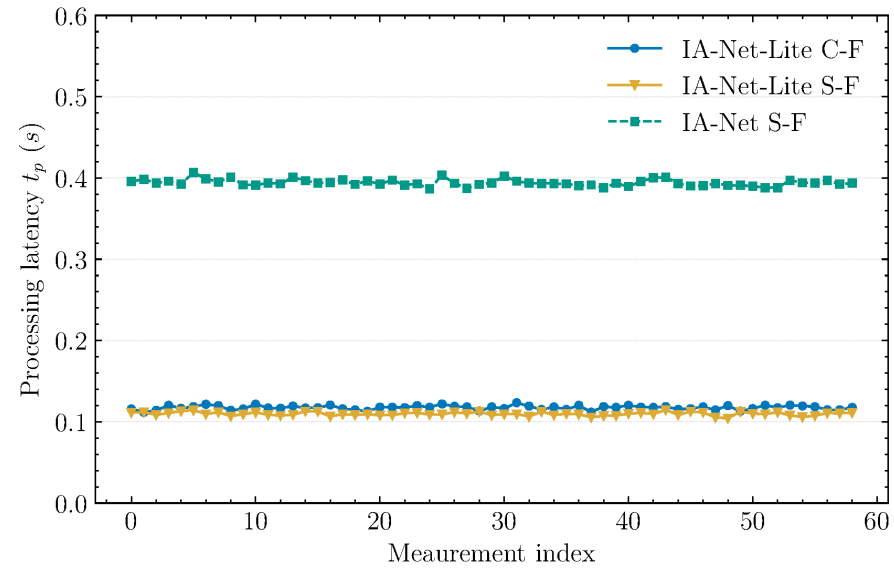

Fig. 6: Overall processing latency in $\mathrm{C}-\mathrm{F}$ and $\mathrm{S}-\mathrm{F}$ modes.

TABLE II: Data throughput and filter rate $r$ on each link, and the total transmission latency $t_{t}$ of IA-Net-Lite in $\mathrm{C}-\mathrm{F}$ and $\mathrm{S}-\mathrm{F}$ modes.

\begin{tabular}{c|c|c|c|c}
\hline \multirow{2}{*}{ Mode } & client $\rightarrow \mathbf{s 1}$ & $\mathbf{s 1} \rightarrow \mathbf{s} 2$ & $\mathbf{s 2} \rightarrow$ server & \multirow{2}{*}{$\mathbf{t}_{\mathbf{t}}$} \\
\hline \multirow{2}{*}{$\mathrm{C}-\mathrm{F}$} & 64397 byte & 40715 byte & 17626 byte & \multirow{2}{*}{$2.1 \mathrm{~s}$} \\
\cline { 2 - 4 } & $25.12 \%$ & $15.88 \%$ & $6.87 \%$ & \\
\hline \multirow{2}{*}{ S-F } & 256406 byte & 256406 byte & 256406 byte & \multirow{2}{*}{$3.2 \mathrm{~s}$} \\
\cline { 2 - 4 } & $100 \%$ & $100 \%$ & $100 \%$ & \\
\hline
\end{tabular}

load required by IA-Net-Lite is reduced to a quarter. Thus, IA-Net-Lite with the lightweight design is more suitable for deployment on resource-constrained network nodes.

Moreover, we observe that IA-Net-Lite s processing latency in $\mathrm{S}-\mathrm{F}$ and $\mathrm{C}-\mathrm{F}$ are different. In $\mathrm{C}-\mathrm{F}$ it is slight about $0.005 \mathrm{~s}$ (5\%) more than that in S-F. Since IA-Net-Lite uses exactly the same processing logic in both $\mathrm{S}-\mathrm{F}$ and $\mathrm{C}-\mathrm{F}$ modes, one of the reasons why this happens could be due to the $\mathrm{I} / \mathrm{O}$ processing delay between the switch and $\mathrm{VNF}$ in $\mathrm{C}-\mathrm{F}$ However, we consider the instability of the processing time to be within the acceptable range compared to the benefit of reduced service latency.

\section{Transmission Latency and Traffic Filter}

To demonstrate the effectiveness of the traffic filter in terms of transmission latency, we listed the data throughput and the filter rate on each network connection in Table [I and gave the results in $\mathrm{S}-\mathrm{F}$ mode for comparison. Since all bandwidths between network nodes in our emulator setup are $10 \mathrm{Mbps}$ with $150 \mathrm{~ms}$ delay, reducing data throughput effectively leads to a decrease in transmission latency.

Table [I shows that after the filtering of VNF on the client and the switch 1 (s1), the bandwidth between them is consuming only $25.12 \%$ and $15.88 \%$ of the original data, respectively, while the high-density information reaching the server is compressed down to $6.87 \%$ of the original data after the last $\mathrm{VNF}$ on the switch 2 (s2). Therefore, the total transmission latency $t_{t}$ in $\mathrm{C}-\mathrm{F}$ mode is reduced to about $2.1 \mathrm{~s}$. This reduction, expressed in the overall service latency, is the gap between IA-Net-Lite with the S-F and C-F mode in Fig. 5
In contrast, the bandwidth consumption of the traditional S-F remains the same as the original data, and thus the transmission latency cannot be reduced. $t_{t}$ stays about $3.2 \mathrm{~s}$ with $\mathrm{S}-\mathrm{F}$ mode. Comparing the $\mathrm{C}-\mathrm{F}$ and $\mathrm{S}-\mathrm{F}$ modes, although they hold exactly the same processing latency, the redundant data is filtered by the VNF; on the network nodes with the help of the in-network processing scheme, thus reducing the transmission latency and finally obtaining a smaller service latency.

\section{CONCLUSION}

Time-critical applications are becoming more and more important in modern smart factories, however, these applications have delayed data transmission and processing due to high-density sensors and large data volumes. In this paper, we show our brand new design for an innetwork data processing with the help of network softwarization for industrial anomaly detection, which we call Information Abstraction Neural Network Lite (IA-Net-Lite). This approach leverages for the first time the resources of increasingly intelligent network devices to combine data transmission and processing with filters that reduce redundancy, and, ultimately, optimize service latency. In this paper, we showed via the employment of a practical network emulator that our proposed approach can reduce the service latency by up to $38.89 \%$. The design idea of this method is also applicable to other large-volume and AI applications.

However, comparing with the theoretical filter rate $r$ in Section III-B the measured $r$ (see Table III) in our emulations is slightly larger. This is due to that the $\mathrm{C}-\mathrm{F}$ can only filter the payloads of the network packets, while the headers remain unchanged. Header compression techniques [18] can be used to reduce the packet headers, thereby maximizing the filtering effect. This is in our future research agenda.

\section{ACKNOWLEDGMENT}

This work was funded by the Federal Ministry of Education and Research of Germany (Software Campus NetBliSS 01IS17044), and by the German Research Foundation (Deutsche Forschungsgemeinschaft) as part of Germany's Excellence Strategy (EXC 2050/1 - Project ID 390696704) Cluster of Excellence "Centre for Tactile Internet with Humanin-the-Loop" (CeTI) of Technische Universität Dresden.

\section{REFERENCES}

[1] H. Wu, J. He, M. Tömösközi, Z. Xiang, and F. H. P. Fitzek, "In-network processing for low-latency industrial anomaly detection in softwarized networks," in 2021 IEEE Global Communications Conference: NextGeneration Networking and Internet (Globecom2021 NGNI), Madrid, Spain, 2021

[2] M. Wollschlaeger, T. Sauter, and J. Jasperneite, "The future of industrial communication: Automation networks in the era of the internet of things and industry 4.0," IEEE Industrial Electronics Magazine, vol. 11, no. 1, pp. 17-27, 2017.

[3] F. Civerchia, S. Bocchino, C. Salvadori, E. Rossi, L. Maggiani, and M. Petracca, "Industrial internet of things monitoring solution for advanced predictive maintenance applications," Journal of Industrial Information Integration, vol. 7, pp. 4-12, 2017. 
[4] V. Chandola, A. Banerjee, and V. Kumar, "Anomaly detection: A survey," ACM Comput. Surv., vol. 41, no. 3, Jul. 2009. [Online]. Available: https://doi.org/10.1145/1541880.1541882

[5] M. H. u. Rehman, E. Ahmed, I. Yaqoob, I. A. T. Hashem, M. Imran, and S. Ahmad, "Big data analytics in industrial iot using a concentric computing model," IEEE Communications Magazine, vol. 56, no. 2, pp. 37-43, 2018.

[6] H. Wu, J. He, M. Tömösközi, and F. H. Fitzek, "Abstraction-based multiobject acoustic anomaly detection for low-complexity big data analysis," in WS17 IEEE ICC 2021 Workshop on Communication, Computing, and Networking in Cyber-Physical Systems (WS17 ICC'21 Workshop - CCNCPS), Montreal, Canada, Jun. 2021.

[7] R. Glebke, M. Henze, K. Wehrle, P. Niemietz, D. Trauth, P. Mattfeld MBA, and T. Bergs, "A case for integrated data processing in large-scale cyber-physical systems," in Proceedings of the 52nd Hawaii International Conference on System Sciences, 2019.

[8] R. Glebke, J. Krude, I. Kunze, J. Rüth, F. Senger, and K. Wehrle, "Towards executing computer vision functionality on programmable network devices," in Proceedings of the 1st ACM CoNEXT Workshop on Emerging in-Network Computing Paradigms, 2019, pp. 15-20.

[9] E. Haleplidis, K. Pentikousis, S. Denazis, J. H. Salim, D. Meyer, and O. Koufopavlou, "Software-defined networking (sdn): Layers and architecture terminology," RFC 7426, 2015.

[10] B. Han, V. Gopalakrishnan, L. Ji, and S. Lee, "Network function virtualization: Challenges and opportunities for innovations," IEEE Communications Magazine, vol. 53, no. 2, pp. 90-97, 2015.

[11] J. Halpern, C. Pignataro et al., "Service function chaining (sfc) architecture," in $R F C$ 7665, 2015.

[12] I. Kunze, K. Wehrle, D. Trossen, and M.-J. Montpetit, "Use Cases for In-Network Computing," Internet Engineering Task Force, Internet-Draft draft-irtf-coinrg-use-cases00, Feb. 2021, work in Progress. [Online]. Available: https://datatracker.ietf.org/doc/html/draft-irtf-coinrg-use-cases-00

[13] M. Sandler, A. Howard, M. Zhu, A. Zhmoginov, and L.-C. Chen, "Mobilenetv2: Inverted residuals and linear bottlenecks," in Proceedings of the IEEE conference on computer vision and pattern recognition, 2018, pp. 4510-4520.

[14] H. Wu, J. He, M. Tömösközi, and F. H. P. Fitzek, "Y-net: A dual path model for high accuracy blind source separation," in 2020 IEEE Globecom Workshops (GC Wkshps), 2020, pp. 1-6.

[15] K. He, X. Zhang, S. Ren, and J. Sun, "Deep residual learning for image recognition," in Proceedings of the IEEE conference on computer vision and pattern recognition, 2016, pp. 770-778.

[16] H. Purohit, R. Tanabe, T. Ichige, T. Endo, Y. Nikaido, K. Suefusa, and Y. Kawaguchi, "MIMII Dataset: Sound dataset for malfunctioning industrial machine investigation and inspection," in Proceedings of the Detection and Classification of Acoustic Scenes and Events 2019 Workshop (DCASE2019), November 2019, pp. 209-213.

[17] Z. Xiang, S. Pandi, J. Cabrera, F. Granelli, P. Seeling, and F. H.-P. Fitzek, "An open source testbed for virtualized communication networks," IEEE Communications Magazine, pp. 1-7, (Accepted, 2020).

[18] M. Tömösközi, D. E. Lucani Rötter, F. H. P. Fitzek, and P. Ekler, "Unidirectional robust header compression for reliable low latency mesh networks," in 2019 IEEE International Conference on Communications (ICC): Mobile and Wireless Networks Symposium (IEEE ICC'19 -MWN Symposium), 2019. 